\title{
Non-Markovian spin relaxation in two-dimensional electron gas.
}

\author{
M.M. Glazov \\ A. F. Ioffe Physico-Technical Institute, Russian Academy of Sciences, 194021 St. Petersburg, Russia \\ E.Ya. Sherman \\ Department of Physics and Institute for Optical Sciences, University of Toronto, \\ 60 St. George Street, Toronto, Ontario, Canada M5S $1 A 7$
}

\begin{abstract}
We analyze by Monte-Carlo simulations and analytically spin dynamics of two-dimensional electron gas (2DEG) interacting with short-range scatterers in nonquantizing magnetic fields. It is shown that the spin dynamics is non-Markovian with the exponential spin relaxation followed by the oscillating tail due to the electrons residing on the closed trajectories. The tail relaxes on a long time scale due to an additional smooth random potential and inelastic processes. The developed analytical theory and Monte-Carlo simulations are in the quantitative agreement with each other.

Journal ref.: Europhys. Lett. 76, pp. 102-108 (2006).
\end{abstract}

Spin-dependent phenomena in semiconductors and semiconductor nanostructures attract an increasing interest during last decade (for review see, e.g. Ref. 1). The understanding of the mechanisms of spin decoherence, usually considered as a Markovian process, as well as the possibilities to control it is an important problem in the field. In the two-dimensional (2D) zinc-blende structures the electron spin dynamics is governed by the spin-orbit (SO) splitting of the conduction band. It originates from the lack of the inversion center either in the bulk material as represented by the bulk inversion asymmetry (BIA or Dresselhaus term 2, 3]) or the structural asymmetry of the heteropotential revealed by the Rashba term [4]. In all the cases the $\mathrm{SO}$ interaction gives rise to the effective Zeeman magnetic field which is characterized by the electron wavevector $\boldsymbol{k}$-dependent spin precession vector $\Omega_{k}$ whose direction determines the axis and the absolute value is the spin precession rate. In the collision dominated regime of electron motion with $\Omega \tau \ll 1$ (where $\Omega$ is the typical value of $\left|\boldsymbol{\Omega}_{\boldsymbol{k}}\right|$ and $\tau$ is the scattering time) the small spin rotation angles $\Omega \tau$ between successive collisions are not correlated, thus the spin relaxation rate $\tau_{s}^{-1}$ is proportional to the $\Omega^{2} \tau$. [3, 5] This D'yakonov-Perel' spin relaxation mechanism, which represents a Markovian process, is the most important one in the wide range of temperatures and carrier concentrations. 1, 6, 7, 8, 9, 10, 11, 12] At given sample parameters there are two possibilities to control the spin relaxation rate with this mechanism. First, one may apply an external electric field in order to tune the Rashba constant [9, 13]. Second, an external magnetic field can suppress the spin relaxation due to the cyclotron rotation, Larmor spin precession or their combination. 14, 15] Although the main mechanisms of the magnetic field effects on the Markovian spin relaxation are understood, [16 the peculiarities in the spin dynamics caused by the fascinating non Markovian transport phenomena in magnetic field, lavished attention only very recently [17, 18, 19], and are scarcely known. Non-Markovian spin dynamics can arise for electrons interacting with nuclear spins 20], hovewer, with a qualitatively different mechanism of the memory.

In this Letter we point out and study a new nonMarkovian effect in electron spin relaxation in the magnetic field, namely, the appearance of the long-living spin polarization tail. We consider a degenerated 2DEG with SO coupling scattered by the short-range impurity centers (or antidots) with the radius $a$ and the concentration $N$ in the presence of the magnetic field $\mathbf{B}$ directed perpendicular to the structure plane. Assuming specular reflection by the scatterers, the kinetics of electrons is characterized by the total scattering length $l=1 /(2 N a)$ and the transport mean free path $l_{t r}=3 l / 4$. As shown in the pioneering work [21] (see also Refs. 22, 23, 24 and references therein) a fraction of electrons $P_{c}(B)$ moving with the velocity $v$ does not experience scattering travelling along the closed circular orbits. In the $N a^{2} \ll 1$ limit it is given by

$$
P_{c}(B)=\exp \left(-2 \pi R_{c} / l\right)
$$

where $R_{c}=v / \omega_{c}$ is the cyclotron radius, $\omega_{c}=e B / m c$ is the cyclotron frequency, $e$ is the elementary charge and $m$ is the electron effective mass. Physically, Eq. (1) gives the probability to find no scatterers in the area between circles of the radii $R_{c} \pm a$, see Fig. 1] As the electron finished the cyclotron revolution without a collision it will stay on this orbit infinitely long, thus demonstrating a non Markovian behavior with an infinitely long memory. The fraction of these electrons increases with the magnetic field resulting in the classical magnetoresistance [23]. Here we discuss the role of the closed orbits in the spin relaxation. We show that the spin dynamics of the electrons on such trajectories is strictly periodical and the total spin falls exponentially down to the value determined by the fraction of circular orbits. Therefore, a tail in the spin polarization can be observed. Further, we develop a theory describing a decay of this tail caused by the scattering processes which transfer electrons between closed and open "wandering" [23] orbits. Finally, 
we discuss the role of the quantum effects.

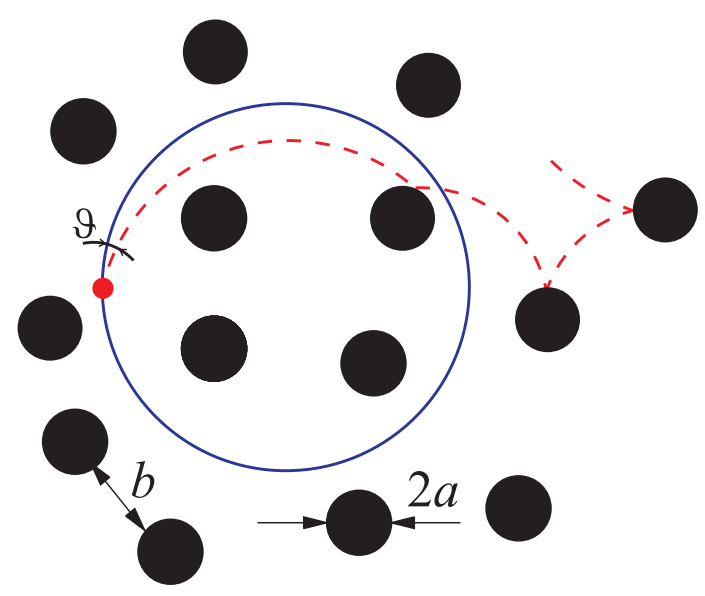

FIG. 1: Electron's motion along the circling trajectory. Black circles - scattering centers. Solid line - initial "circling" trajectory. Dashed line - wandering trajectory after the inelastic scattering event with the scattering angle $\vartheta$. Here $a$ is the scatterer radius and $b \sim N^{-1 / 2}$ is the mean distance between scatterers. Figure shown not to scale.

In order to obtain quantitative results we consider a $\hat{z} \|$ [001]-grown zince-blende quantum well (QW) and assume that the SO interaction is dominated by the Dresselhaus term [3]

$$
\mathcal{H}_{S O}(\boldsymbol{k})=\frac{\hbar}{2}\left(\boldsymbol{\sigma} \cdot \boldsymbol{\Omega}_{\boldsymbol{k}}\right), \quad \boldsymbol{\Omega}_{\boldsymbol{k}}=\Omega_{0}\left[\cos \varphi_{\boldsymbol{k}},-\sin \varphi_{\boldsymbol{k}}, 0\right]
$$

where $\boldsymbol{\sigma}=\left(\sigma_{x}, \sigma_{y}, \sigma_{z}\right)$ is the Pauli matrix vector, $\Omega_{0}$ is the spin precession frequency at the Fermi level and $\varphi_{\boldsymbol{k}}$ is the angle between electron wavevector $\boldsymbol{k}$ and $\hat{x} \|$ [100] axis.

We assume classical dynamics of the electrons and their spins and subdivide the electrons into two groups. First one corresponds to wandering electrons while the second one corresponds to circling electrons, their relative fractions are $1-P_{c}(B)$ and $P_{c}(B)$, respectively. The accurate description of the wandering electrons in the moderate magnetic fields can be done by using the kinetic equation and their spin decays exponentially with the rate [14]

$$
\frac{1}{\tau_{s}(B)}=\frac{\Omega_{0}^{2} \tau}{1+\left(\omega_{c} \tau\right)^{2}}
$$

where $\tau=l_{t r} / v_{F}$. In the derivation of Eq. (3) we assumed $\left.\Omega_{0} \tau \ll 1 \quad{ }^{1}\right)$ and neglected the Larmor effect on spin relaxation as it is usually small in GaAs-based QWs. The Larmor effect can be taken into account in a standard way. 16.

\footnotetext{
1 This condition can be relaxed provided $\Omega_{0} \ll \omega_{c}$, M.M. Glazov, to be published
}

The spin of the single electron with $\boldsymbol{k}(t)$-dependent wavevector is described by equation

$$
\frac{\partial s}{\partial t}+s \times \boldsymbol{\Omega}_{\boldsymbol{k}(t)}=0
$$

Eq. (4) can be readily solved assuming that $\boldsymbol{k}$ rotates in the QW plane with the frequency $\omega_{c}$. For the initial condition $2 \boldsymbol{s}(0)=(0,0,1)$, we obtain for $z$-component

$$
2 s_{z}(t)=\frac{\omega_{c}^{2}}{\omega_{c}^{2}+\Omega_{0}^{2}}+\frac{\Omega_{0}^{2}}{\omega_{c}^{2}+\Omega_{0}^{2}} \cos \left(\sqrt{\Omega_{0}^{2}+\omega_{c}^{2}} t\right) .
$$

The solutions for $s_{x, y}$ are rather cumbersome and not presented here. It is seen in Eq. (5) that on the circling trajectory $2 s_{z}$ oscillates around the mean value $\omega_{c}^{2} /\left(\omega_{c}^{2}+\Omega_{0}^{2}\right) \rightarrow 1$ (in high fields, $\left.\omega_{c} \gg \Omega_{0}\right)$ with the frequency $\sqrt{\Omega_{0}^{2}+\omega_{c}^{2}}$, and the amplitude of the oscillations is $\Omega_{0}^{2} /\left(\omega_{c}^{2}+\Omega_{0}^{2}\right) \rightarrow 0$ at $\omega_{c} \gg \Omega_{0}$. We note that these oscillations are insensitive to the initial phase of the $k$ rotation and thus all the spins of the circling electrons oscillate synchronously.

Therefore, if in the 2DEG the transitions between circling and wandering trajectories are strictly forbidden, at $t \gg \tau_{s}(B)$ (defined by Eq. (3)) the total spin does not decay to zero as it was assumed previously, but oscillates around the non-zero value

$$
s_{z}^{t}=s_{0} P_{c}(B) \frac{\omega_{c}^{2}}{\omega_{c}^{2}+\Omega_{0}^{2}},
$$

where $s_{0}$ is the initially excited spin. Further we consider the case of the strong enough magnetic fields, $\omega_{c} \gg \Omega_{0}$ where the oscillations of the circling electron spin are small and the spin relaxation is followed by the nonvanishing tail with the magnitude $s_{z}^{t}=P_{c}(B) s_{0}$. In order to have an insight into the tail formation we performed a Monte-Carlo simulation of the electron transport and spin dynamics with the results shown in Fig. 2 It is seen that the exponential decay of the velocity autocorrelation function $K_{v v}(t)=\langle\mathbf{v}(t) \mathbf{v}(0)\rangle / v^{2}$ (shown in the inset) is followed by the oscillations due to the circling electrons. The magnitude of the oscillations corresponds to the value of $P_{c}(B)$. The polarization exhibits oscillatory tail in agreement with Eqs. (5), (6). We mention that in nonquantizing field $B=0.5 \mathrm{~T}$ the tail has a considerable magnitude $P_{c}(B)>0.1$ already at low mobilities $\mu=5 \times 10^{4} \mathrm{~cm}^{2} / \mathrm{Vs}$.

Now we turn to the decay of the spin polarization tail. Any additional scattering process such as inelastic scattering or scattering by a random potential destroys the tail. With the relaxation time approximation the $z$ spin component is described by the coupled kinetic equations:

$$
\begin{aligned}
\frac{\partial s_{z}^{c}}{\partial t} & =-\frac{s_{z}^{c}\left[1-P_{c}(B)\right]-s_{z}^{w} P_{c}(B)}{\tau_{j}(B)} \\
\frac{\partial s_{z}^{w}}{\partial t} & =\frac{s_{z}^{c}\left[1-P_{c}(B)\right]-s_{z}^{w} P_{c}(B)}{\tau_{j}(B)}-\frac{s_{z}^{w}}{\tau_{s}(B)} .
\end{aligned}
$$



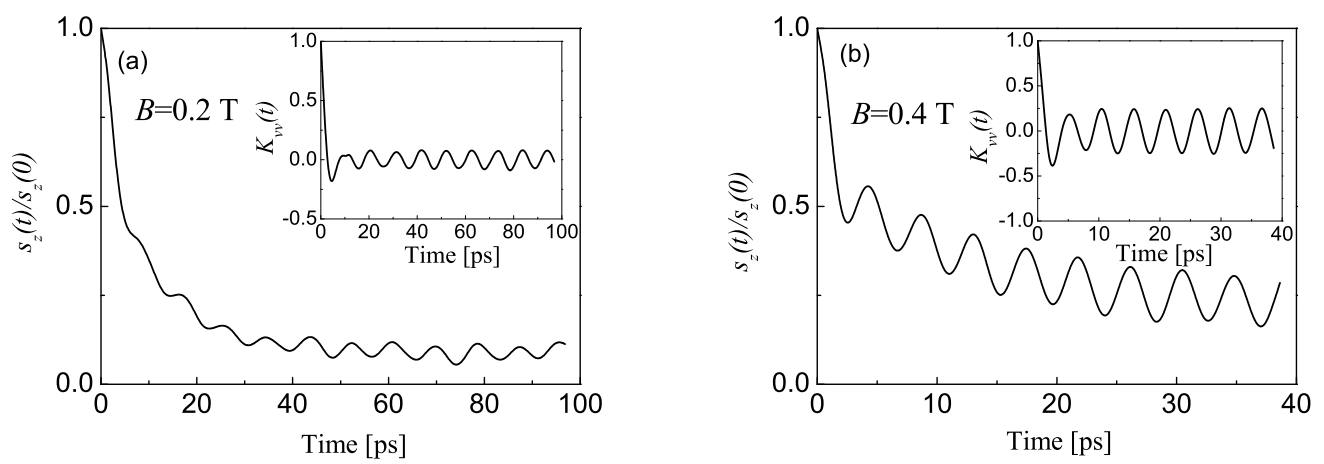

FIG. 2: The results of the Monte-Carlo simulation for 4096 electrons of the spin dynamics $s_{z}(t) / s_{z}(0)$ and the velocity autocorrelation function $K_{v v}(t)$. The scatterer radius is $a=2 \times 10^{-6} \mathrm{~cm}, v=2 \times 10^{7} \mathrm{~cm} / \mathrm{s}, N=3 \times 10^{9} \mathrm{~cm}^{-2}$, and $B=0.2 \mathrm{~T}$, $\Omega_{0}=4 \times 10^{11} \mathrm{~s}^{-1}\left[\right.$ panel (a)] and $B=0.4 \mathrm{~T}, \Omega_{0}=8 \times 10^{11} \mathrm{~s}^{-1}\left[\right.$ panel (b)]; $\omega_{c}=3.0 \times 10^{12} \mathrm{~s}^{-1} / \mathrm{T}$.

Here superscript $c(w)$ corresponds to circling (wandering) electrons, respectively, and $\tau_{s}(B)$ and $\tau_{j}(B)$ is the spin relaxation time for wandering electrons and the intermixing time between the closed and wandering trajectories. The first term in the numerator of the right hand side of the Eqs. (77) denotes the transfer of the electron from the circling to the wandering trajectory and is proportional to the scattering intermixing rate and the fraction of the wandering trajectories $1-P_{c}(B)$ and the second term refers to the inverse process. In deriving Eqs. (77) we have neglected small oscillations of $s_{z}^{c}$ and took into account D'yakonov-Perel' spin relaxation of the wandering electrons (second term of the second line in (17). The eigenrates of the system $\tau_{1,2}^{-1}(B)$ are

$$
\frac{1}{\tau_{1,2}(B)}=\frac{1}{2}\left(\Gamma_{j s} \pm \sqrt{\Gamma_{j s}^{2}+4 \frac{P_{c}(B)-1}{\tau_{j}(B) \tau_{s}(B)}}\right),
$$

where $\Gamma_{j s}=\tau_{j}^{-1}(B)+\tau_{s}^{-1}(B)$. Thus, the total spin of electrons $s_{z}(t)$ relaxes according to the two exponential law: $s_{z}(t)=\alpha \exp \left(-t / \tau_{1}(B)\right)+\beta \exp \left(-t / \tau_{2}(B)\right)$ where coefficients $\alpha$ and $\beta$ depend on the initial condition and $B$. Since $\tau_{2}(B) \geq \tau_{1}(B)$ long-time $s_{z}(t)$ asymptotics is determined by $\tau_{2}(B)$ which can be identified with the tail relaxation time.

For example, in the limit of weak magnetic fields $P_{c}(B) \ll 1$ and if $\tau_{s}(B) \ll \tau_{j}(B)$ the relaxation times are $\tau_{1}(B)=\tau_{s}(B)$ and $\tau_{2}(B)=\tau_{j}(B)$ and the total electron spin rapidly decays to the tail value $P_{c}(B) s_{0}$ and then slowly relaxes during the time $\tau_{j}(B)$ (as almost every "additional" scattering transfers electron from the circling to wandering trajectory where the spin is quickly lost).

When the magnetic field becomes stronger with $\omega_{c} \tau \gg$ $1, \tau_{s}(B)=\tau_{s}(0)\left(\omega_{c} \tau\right)^{2}$ increases quadratically with the field and the fraction $P_{c}(B)=1-3 \pi / 2 \omega_{c} \tau$ tends to unity. Two limiting regimes as a function of $B$ are possible. In weak fields, where the intermixing $\tau_{j}$ is still much longer than $\tau_{s}(B)$, the spin tail relaxation time is field independent. With the increase of $B$, the intermixing time becomes smaller than $\tau_{s}(B)$, and, therefore, the tail disappears. In such a case only a small fraction $\left(\sim \omega_{c} \tau\right)$ of electrons is loosing at a given moment their spin, however circling and wandering electrons are intermixed on a short timescale. Therefore the tail is not observed but the spin relaxation time is:

$$
\tau_{2}(B) \approx \frac{l}{2 \pi R_{c}} \tau_{s}(B) \approx \frac{2\left(\omega_{c} \tau\right)^{3}}{3 \pi \Omega_{0}^{2} \tau}
$$

being proportional to $B^{3}$ (contrary to the standard result $B^{2}[14)$ ). Here $\tau_{2}(B)$ does not depend on the inelastic scattering time despite the presence of the strong inelastic scattering which is necessary here only to intermix the trajectories sufficiently quickly. These results are shown in Fig. 3 illustrating the evolution of the spin relaxation process $s_{z}(t)$ and the rate with the change in the number of wandering trajectories governed by the $B$-dependent ratio $l / R_{c}$.

In sufficiently strong fields, the number of trajectories colliding several times with the same impurity, increases, and at a critical field such as $4 \pi N\left(a+R_{c}\right)^{2} \approx 4.4$ and $\left(R_{c} / l\right)^{2}=4.4 N a^{2} / \pi \ll 1[22]$ all the paths become confined. For the parameters considered in Fig.(2), this corresponds to $B>0.8 \mathrm{~T}$. In this case the spin relaxation is strongly suppressed [25]. The detailed analysis of this regime is beyond the scope of this Letter.

Below, we consider two kinds of the microscopic processes which contribute to the intermixing time $\tau_{j}(B)$ between closed and wandering trajectories. Namely, we discuss the destruction of the tail due to: (i) presence of the smooth potential, (ii) inelastic processes.

(i) In the real QWs the typical disorder can be considered as the superposition of the short-range scattering 

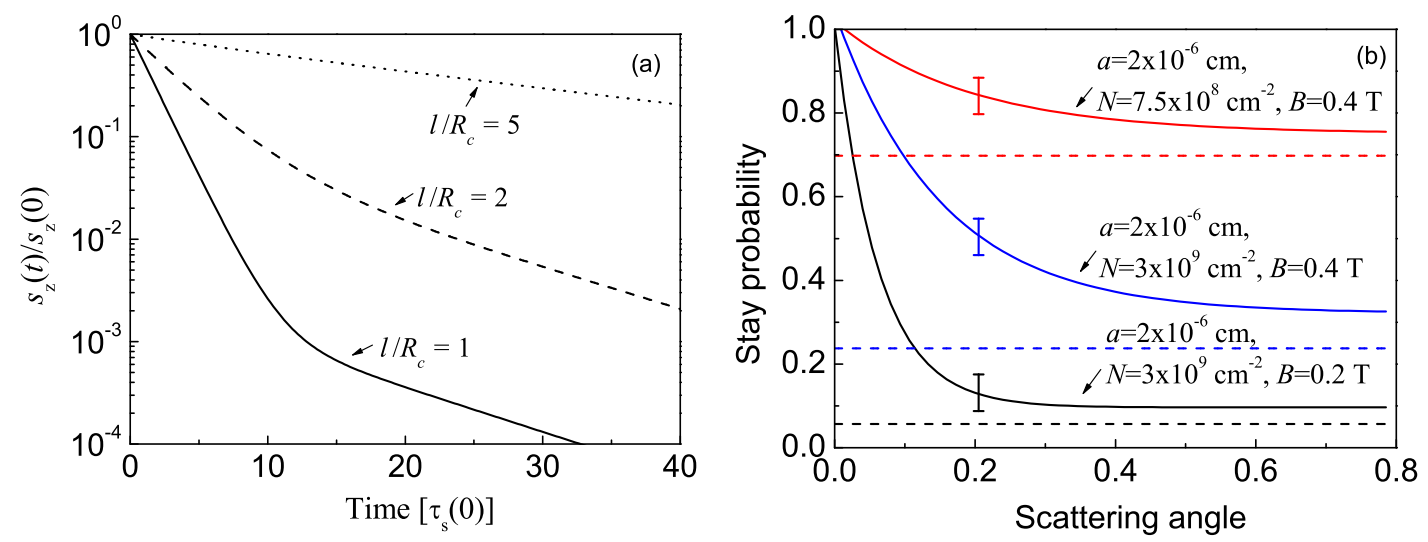

FIG. 3: (a) Electron spin $s_{z}$ time dependence calculated from Eqs. (7) for the different values of the magnetic field: $l / R_{c}=1,2,5$ from the bottom to the top, $\tau_{j}=10 \tau_{s}(B=0)$. (b) The scattering probability found by the Monte-Carlo simulation for (solid lines) and values of $P_{c}(B)$ (dashed). The parameters used in the calculations are shown above each curve. Other parameters are same as in Fig.(2). The accuracy of the Monte-Carlo computation is shown by the error bars.

centers $\left({ }^{2}\right)$ and the long-range smooth potential of the remote dopants and interface roughness $\left({ }^{3}\right)$. The correlation radius of this potential $d$ is the distance between the dopant layer and the QW. 26, 27] We will be interested in the weak smooth disorder where the transport scattering time by long-range potential $\tau_{l}$ satisfies the condition $\tau_{l} \omega_{c} \gg 1$, and, therefore, the circling trajectories remain at least approximately intact. The displacement of the orbit center in the smooth potential provided $R_{c} \gg d$ is $\delta r \sim l_{l} /\left(\omega_{c} \tau_{l}\right)^{3 / 2} \sim \sqrt{R_{c}^{3} / d}\left(W / E_{F}\right)$, where $l_{l}=v_{F}$ $\tau_{l}$ is the transport mean free path there, $W$ is the potential amplitude. 28 The transfer between trajectories is governed by the ratio $\delta r / a$. The rich transport 26] and spin dynamics of the electrons in the system with two types of disorder are beyond the scope of this Letter. Here we consider the simplest situation where $\delta r \ll a$ and thus $\delta r \ll d$. Therefore, electron drifts in the potential gradient averaged over its path and sweeps during $n$ revolutions an area $S \sim 2 \pi R_{c} n \delta r$. It will be transferred from the circling trajectory to the wandering as soon as it meets a short-range center in this area, i.e. $S N \sim 1$. So, the intermixing time can be estimated as

$$
\tau_{j}^{s p}(B) \sim \frac{T_{c}}{N R_{c} \delta r} \propto B^{3 / 2}, \quad \delta r / a \ll 1
$$

where $T_{c}=2 \pi / \omega_{c}$ is the cyclotron period and it is assumed that $a l_{t r} /\left(d R_{c}\right) \ll 1$. For the purpose of the present paper we note that the electron drift in the

\footnotetext{
2 These centers can be introduced artificially as antidotes, see e.g. 17, 26, 32

3 The monolayer fluctuations of the QW interfaces induce the potential $\delta V \sim 1 \ldots 10 \mathrm{meV}$ with the lateral size $50 \ldots 100 \AA$. Thus, the scattering length on the interface roughnesses $a$ is of the order of $10^{4} \AA$
}

smooth potential leads to the spin relaxation even for the circular trajectories, therefore the applicability of the Eq. (10) together with the system (7) is guaranteed provided if the intermixing time is much longer than the spin relaxation time of the circling electrons.

(ii) The inelastic processes (electron-electron or electron-phonon scattering) change randomly electron wavevector and energy and thus transfer electrons between circling and wandering trajectories. In the low temperature regime where the Fermi surface is well defined, $E_{F} \gg k_{B} T$, where $E_{F}$ is the Fermi energy and $k_{B} T$ is the temperature measured in the energy units all the scattering processes are statistically quasi-elastic [29], i.e. the transferred energy $\sim k_{B} T$ is much smaller than electron kinetic energy $E_{F}$. The crucial question is to determine the probability that the scattering at the angle $\vartheta$ (Fig. 1) is not accompanied by the electron transition from the circling to wandering orbit. From Fig. [1] it is clear that the displacement of the circular orbit by the distance of the order of the impurity separation $b$ will lead to the electron collision with some scattering center thus the probability should have a peak in the vicinity of $\vartheta=0$ with the angular width of the order of $b / R_{c} \sim 1 /\left(N^{1 / 2} R_{c}\right)$. At larger angles the probability saturates at the value close to $P_{c}(B)$ representing the fraction of the circling electrons. This prediction is corroborated by the Monte-Carlo calculation, see Fig. 3 b.

In a degenerate $2 \mathrm{D}$ electron gas with carrier concentration ranging in $10^{11} \ldots 10^{12} \mathrm{~cm}^{-2}$ the Coulomb interaction is screened and the electron-electron scattering is almost isotropic and its temperature dependency is determined by the Pauli-exclusion principle only. At not too low temperatures $k_{B} T \gtrsim \hbar c_{s} \sqrt{\left\langle k_{z}^{2}\right\rangle}$ where $c_{s}$ is the speed of sound, and $\sqrt{\left\langle k_{z}^{2}\right\rangle}$ is the root mean square of the electron wavevector in the growth direction, the electronacoustic phonon scattering is dominated by deformation- 
potential coupling and is isotropic as well. Thus we further assume that the inelastic scattering is isotropic and can be described in the framework of the relaxation time $\tau_{j}$ which can be estimated as for electron-electron and electron-phonon scattering, respectively [30, 31]:

$$
\tau_{j}^{e e}=\zeta_{e} \frac{\hbar}{E_{F}}\left(\frac{E_{F}}{k_{B} T}\right)^{2}, \quad \tau_{j}^{p h}=\zeta_{p h} \frac{\rho c_{s}^{2} \hbar^{3}}{D^{2} m k_{B} T \sqrt{\left\langle k_{z}^{2}\right\rangle}} .
$$

Here $\zeta_{e}=4 /\left[\pi \ln \left(E_{F} / k_{B} T\right)\right]$ for Fermi level electrons, $D$ is the deformation potential constant, $\rho$ is the material density, and $\zeta_{p h} \sim 1$ is the numerical coefficient. At lower temperatures the electron-phonon scattering is partially suppressed due to Pauli-exclusion principle and by the phonon-freeze-out and the coefficient $\zeta_{p h}$ becomes strongly temperature dependent. Its exact calculation represents an extremely difficult numerical tasks but the simple estimates show that in the temperature range $T \leq 20 \mathrm{~K}$ and for electron concentration $N_{e} \sim 10^{11}$ $\mathrm{cm}^{-2}$ the electron-electron scattering is dominant. We note that the fraction of the circling electrons $P_{c}(B) \mathrm{Eq}$. (11) has a purely geometric character, thus the tail is insensitive to the thermal broadening of the electron distribution provided inelastic processes are weak enough.

Finally, we would like to comment on the quantum effects neglected above. First, the quantization of electron orbits leads to the oscillatory $B$-dependence of the spin relaxation rate (3) 33]. This effect is small for high Landau levels $\left(E_{F} / \hbar \omega_{c} \gg 1\right)$ and vanishes as temperature increases. Second, the quantization of the electron orbits modifies the relaxation rates of inelastic scattering in Eq. (11). However, for semiclassical Landau levels this modification is negligible 29]. Third, the magnetic field leads to the spin polarization either due to Zeeman effect or as a result of the SO coupling and Landau quantization [4]. The former contribution is proportional to $g \mu_{B} B / E_{F}$ where $g$ is the electron $g$-factor and $\mu_{B}$ is Bohr magnetron. For the typical GaAs structures $g$ is small, besides it can be adjusted to zero with the structure parameters [6]. The contribution $\left|\mathcal{P}_{L}\right|=\left|\left\langle\psi_{L, \pm}\left|\sigma_{z}\right| \psi_{L, \pm}\right\rangle\right|$ of the latter effect can be evaluated for $L$ th Landau level $E_{L, \pm}=\hbar \omega_{c}\left(L \pm \sqrt{\gamma^{2} L+1 / 4}\right)$ with $\gamma=\hbar \Omega_{0} /\left(2 \sqrt{E_{F} \hbar \omega_{c}}\right)$ as $\left|\mathcal{P}_{L}\right|=\left(2 \sqrt{\gamma^{2} L+1 / 4}\right)^{-1}$ and is negligible for high Landau leves with $\gamma^{2} L \gg 1$.

In conclusion, we have theoretically analyzed the spin dynamics of the 2D electron gas scattered by short-range defects in the classically strong magnetic fields. We have shown that the tail in the spin polarization appears as a result of the collisionless circular motion of the fraction of electrons. It was demonstrated that the tail decays due to the presence of the additional weak long-range potential or due to inelastic scattering. In strong magnetic fields the long-time spin relaxation is slowed down as a cube of the field. The predicted phenomena open new perspectives in the spin dynamics control in the 2DEG.

The authors are grateful to E.L. Ivchenko and J.E.
Sipe for the valuable discussions. M.M.G. acknowledges the financial support from RFBR, programs of RAS and "Dynasty" foundation - ICFPM. E.Y.S. was supported by DARPA SpinS program and the FWF (Austria) grant P15520.

[1] Zutic I., Fabian J., and Sarma S. D., Rev. Mod. Phys. 76 (2004) 323.

[2] Dresselhaus G., Phys. Rev. 100 (1955) 580.

[3] Dyakonov M. and Kachorovskii V., Sov. Phys. Semicond. 20 (1986) 110.

[4] Rashba E.I., Sov. Phys. Solid State 2 (1964) 1874.

[5] Dyakonov M. and Perel' V., Sov. Phys. Solid State 13 (1972) 3023.

[6] E. L. Ivchenko, Optical Spectroscopy of Semiconductor Nanostructures (Alpha Science), 2005.

[7] Averkiev N., Golub L., and Willander M., J. Phys.: Condens. Matter 14 (2002) R271.

[8] Malinowski A., Britton R. S., Grevatt T., Harley R. T., Ritchie D. A., and Simmons M. Y., Phys. Rev. B (2000) 13034.

[9] Karimov O., John G., Harley R., Lau W., Flatte M., Henini M., and Airey R., Phys. Rev. Lett. 91 (2003) 246601.

[10] Brand M. A., Malinowski A., Karimov O. Z., Marsden P. A., Harley R. T., Shields A. J., Sanvitto D., Ritchie D. A., and Simmons M. Y., Phys. Rev. Lett. 89 (2002) 236601.

[11] Song P. H. and Kim K. W., Phys. Rev. B 66 (2002) 035207.

[12] Ohno Y., Terauchi R., Adachi T., Matsukura F., and Ohno H., Phys. Rev. Lett. 83 (1999) 4196.

[13] Miller J. B., Zumbuhl D., Marcus C., LyandaGeller Y. B., Goldhaber-Gordon D., Campman K., and Gossard A. C., Phys. Rev. Lett. 90 (2003) 076807.

[14] Ivchenko E. L., Sov. Phys. Solid State 15 (1973) 1048. Ivchenko E. L., Kop'ev P. S., Kochereshko V. P., Uralstev I. N., Yakovlev D. R., JETP Lett. 47 (1988) 486.

[15] Wilamowski Z. and Jantsch W., Phys. Rev. B 69 (2004) 035328.

[16] Glazov M. M., Phys. Rev. B 70 (2004) 195314.

[17] Y. V. Pershin and V. Privman, Phys. Rev. B 69, 073310 (2004).

[18] Lyubinskiy I. S. and Kachorovskii V., Phys. Rev. B 70 (2004) 205335. Lyubinskiy I. S. and Kachorovskii V. Y., Phys. Rev. B $\mathbf{7 3}$ (2006) 041301.

[19] Glazov M. M. and Sherman E. Y., Phys. Rev. B 71 (2005) 241312.

[20] Merkulov I. A. , Efros Al. L, Rosen M., Phys. Rev. B 65 (2002) 205309. Khaetskii A., Loss D., Glazman L., Phys. Rev. B 67 (2003) 195329.

[21] Baskin E., Magarill L., and Entin M., JETP 48 (1978) 365.

[22] Baskin E. and Entin M., Physica B 249 (1998) 805.

[23] Dmitriev A., Dyakonov M., and Jullien R., Phys. Rev. Lett. 89 (2002) 266804.

[24] Bobylev A. V., Maao F. A., Hansen A., and Hauge E. H., J. Stat. Phys. 87 (1997) 1205.

[25] Lyubinskiy I. S., JETP Letters 83 (2006) 336.

[26] Polyakov D. G., Evers F., Mirlin A. D., and Wölfle P., 
Phys. Rev. B 64 (2001) 205306.

[27] Mirlin A. D., Polyakov D. G., Evers F., and Wölfle P., Phys. Rev. Lett. 87 (2001) 126805.

[28] Mirlin A. D., Wilke J., Evers F., Polyakov D. G., and Wölfle P., Phys. Rev. Lett. 83 (1999) 2801.

[29] Gantmakher V. and Levinson Y., Carrier Scattering in Metals and Semiconductors (North-Holl.), 1987.

[30] Glazov M. and Ivchenko E., JETP 99 (2004) 1279.
[31] Price P. J., Annals of Physics 133 (1981) 217.

[32] Sotomayor Choque N. M., Gusev G. M., Leite J. R., Bykov A. A., Litvin L. V., Moshegov N. T., Toropov A. I., Maude D. K., Portal J. C., Phys. Rev. B 66 (2002) 035324.

[33] Burkov A. A., Balents Leon, Phys. Rev. B 69 (2004) 245312. 